\title{
Abstracts from the ASENT 2009 Annual Meeting March 5-7, 2009
}

\section{Abstract \#1: Cognitive Function Data in Newly Diagnosed AED-Naïve Epilepsy Patients Across a Broad Age Range}

Patrick Turk,* Brian K. Saxby,* Mark Bardwell,* Chris Edgar, ${ }^{\dagger}$ and Keith Wesnes*

* Cognitive Drug Research Ltd; ${ }^{\dagger}$ Clinical Trials Consultant

Introduction: Recent initiatives have resulted in guidelines for the assessment of cognitive function in specific clinical populations including the MATRICS consensus cognitive test battery for schizophrenia and the EMEA guideline on medicinal products for the treatment of Alzheimer s disease and other dementias. There are a number of common criteria that emerge, including assessment of cognition at the level of individual cognitive domains; validation; utility; utility as a repeated measure; and data allowing interpretation of results and understanding of the clinical relevance (i.e., normative/relationship to functional outcomes). It is apparent that such common criteria could usefully be applied to cognitive function assessment in all clinical populations. The publication of normative data is of use in the interpretation of clinical trials data.

Methods: Five-hundred and seventy (570) patients with newly diagnosed epilepsy, aged 12-75 years, from 21 countries within Europe, Latin America, and Australasia, not previously exposed to AEDs, were recruited to a clinical trial comparing the efficacy and safety of remacemide versus carbamazepine. Patients completed a 30 minute cognitive test battery comprised of the CDR System; Rey auditory verbal learning test; color-word Stroop test; symbol digit modalities test; Lafayette grooved pegboard; digit cancellation test; and the non-verbal matrices subtest of the Kaufman brief intelligence test. Normative data in treatment-naïve patients across the studied age range were generated.

Results: Assessments showed a common relationship with age, with those patients in the younger adult age cohorts tending to perform better on all measures than older patients and also than the youngest age cohort patients.

Conclusions: These data may be useful in the analysis and interpretation of cognitive assessment data from clinical trials of AEDs and/or studies in similar patient populations.

Financial disclosure: This study was sponsored by AstraZeneca. None of the authors has a financial relationship with the sponsor.

\section{Abstract \#2: Adults with Ornithine Transcarbamylase Deficiency (OTCD) Demonstrate Persistent Abnormality in Cerebral Glutamate Metabolism}

Andrea L. Gropman, ${ }^{*}$ Napapon Sailasuta, ${ }^{\dagger}$ Kent Harris, ${ }^{\dagger}$ Brian Ross, ${ }^{\dagger}$ and Osama Abulseoud

*Department of Neurology, Children's National Medical Center, Washington, DC; ${ }^{\dagger}$ Huntington Medical Research

Institute, Pasadena, CA; ${ }^{\star}$ Department of Psychiatry, Keck School of Medicine, USC, Los Angeles, CA

Objective: 13C MRS to determine cerebral glutamate turnover rate in partial ornithine transcarbamylase deficiency (OTCD)
Background: Patients with OTCD manifest neuropsychological disorders. Proton magnetic resonance spectroscopy $(1 \mathrm{H}$ MRS) demonstrates neurochemical abnormalities in OTCD similar to chronic and subclinical hepatic encephalopathy (SCHE) in whom impaired cerebral glucose metabolism and reduced glutamine-glutamate cycling or glutamate neurotransmission rate (GNT) have been observed. We hypothesize impaired GNT may underlie cognitive deficits in OTCD.

Design/Methods: MRI, $1 \mathrm{H}$ MRS and 13C MRS were performed on a clinical GE $1.5 \mathrm{~T}$ MR scanner in 6 patients with OTCD and 4 normal controls, imaged in stable condition. Each received i.v. $13 \mathrm{C}$ glucose $(0.2 \mathrm{~g} / \mathrm{kg}) \mathrm{C} 1$ or $\mathrm{C} 2$, as a $15-\mathrm{min}$ bolus. Cerebral metabolites were determined with proton decoupling, in posterior cingulate gyrus (PCG, $\mathrm{N}=9$ ) and without proton decoupling, in anterior $(\mathrm{N}=1)$ cingulate gyrus (ACG) over 60-100 minutes.

Results: 1. Uptake and removal of cerebral glucose (13C-C1 or C2) were comparable in normal controls and subjects with OTCD $(p>0.1)$. 2. Glucose $\mathrm{C} 1$ was metabolized to glutamate $\mathrm{C} 4$ and glucose $\mathrm{C} 2$ to glutamate $\mathrm{C} 5$ at comparable rates which were significantly reduced in OTCD (combined $p<0.04$ ). 3. No significant differences in glutamine formation were found in OTCD $(p>0.1)$. 4. 13C - 2 glucose and its metabolic products were observed in ACG without proton decoupling in a single subject with OTCD.

Conclusions/Relevance: Reduced GNT secondary to impaired cerebral glucose metabolism was documented in OTCD using 13C MRS of occipital lobe. Feasibility of this measurement in more relevant frontal brain structures was demonstrated. Treatment(s) which improve cerebral glucose metabolism and GNT may improve neurological outcome in OTCD where prevention and treatment of hyperammonemic episodes appears insufficient.

\section{Abstract \#3: APP Secretases As Therapeutic Targets for Traumatic Brain Injury}

David J. Loane,* Yasuji Matsuoka, ${ }^{\dagger}$ G. William Rebeck,* Alan I. Faden,* and Mark P. Burns*

*Department of Neuroscience; ${ }^{\dagger}$ Department of Neurology, Georgetown University, Washington, DC

Traumatic brain injury (TBI) is a significant risk factor for the development of Alzheimer's disease, and post mortem studies show that $30 \%$ of TBI fatalities have amyloid ${ }^{2}\left(\mathrm{~A}^{2}\right)$ deposits. Remarkably, these deposits occur less than one day after injury. Not only does $\mathrm{A}^{2}$ accumulate after TBI, but so do the necessary amyloid precursor protein (APP) enzymes responsible for $\mathrm{A}^{2}$ production: BACE1 protein $\left({ }^{2}\right.$-secretase) and the gamma-secretase complex protein presenilin-1 (PS-1). In order to understand the role of these secretases in brain trauma, we targeted ${ }^{2}$ - and gamma-secretases with genetic knockout (BACE1-/-) and pharmacological inhibition (DAPT treatment) in an experimental model of TBI.

We exposed WT and BACE1-/- mice and vehicle- and DAPT-treated C57/B16 mice to the controlled cortical impact model of TBI. In this model, an anesthetized animal has a $4 \mathrm{~mm}$ craniotomy performed on the central aspect of the left parietal 
bone. The impounder tip of the pneumatic injury device impacts the cortical surface at a moderate level $(8.0 \mathrm{~m} / \mathrm{s}$ velocity, $2 \mathrm{~mm}$ tissue deformation). This model induces both necrotic and apoptotic cell death, causing a brain lesion, and results in the development of behavioral deficits.

Exposure to TBI resulted in accumulation of endogenous mouse $\mathrm{A}^{2}$ within 1 day, with $\mathrm{A}^{2}$ levels increased by almost $120 \%$ at day 3 before normalizing by day 7 . As expected, the accumulation of $\mathrm{A}^{2}$ corresponded with increased protein levels of APP, BACE1, and PS-1. We found that BACE1-/- and DAPT-treated mice had reduced motor deficits and decreased spatial memory impairments following trauma when compared to WT and Vehicle-treated mice. T2-weighted MRI analysis and histological assessment revealed that $\mathrm{BACE}-/-$ and DAPT treatment spared large areas of cortex and subcortical white matter tracks, reduced CA1 hippocampal neuron loss, and significantly reduced lesion size by $30 \%$ and $>70 \%$, respectively. Furthermore, DAPT treatment reduced endogenous $\mathrm{A}^{2}$ levels by $25 \%$ following trauma.

These data demonstrate that the APP secretases are important mediators of tissue loss following TBI, and that blocking either ${ }^{2}$ - or gamma-secretases can ameliorate motor and cognitive deficits and reduce cell loss following experimental TBI. Thus, modulation of APP secretases may provide novel therapeutic targets for the treatment of TBI.

\section{Abstract \#4: Therapeutic Effect of 1-Octanol in Essential Tremor Likely Mediated Through Octanoic Acid: Data from a High-Dose Extension Study \\ Dietrich Haubenberger, ${ }^{*}$ Fatta Nahab, ${ }^{*}$ Camilo Toro,* Loretta Wittevrongel,* Codrin Lungu,* Mark Hallett,* and Jack A. McLane ${ }^{\dagger}$ \\ *Human Motor Control Section, NINDS, NIH; ${ }^{\dagger}$ Ariston Pharmaceuticals, Inc.}

Background: The therapeutic efficacy of the eight-chain alcohol oral 1-octanol in adults with essential tremor has been demonstrated in doses up to $64 \mathrm{mg} / \mathrm{kg}$ (Shill, 2004). A phase-2 clinical trial assessing pharmacokinetic properties of 1-octanol revealed a dose-dependent plasma response of octanoic acid (OA), while 1-octanol was only detectable at very low plasma levels (clinicaltrials.gov NCT00102596). To further assess the clinical and pharmacokinetic properties of 1-octanol, we escalated the dose in two patients to $128 \mathrm{mg} / \mathrm{kg}$.

Methods: Two right-handed patients (1 f, $1 \mathrm{~m})$, who participated in the $64 \mathrm{mg} / \mathrm{kg}$ fixed-dose study phase, received oral 1 -octanol at a dose of $128 \mathrm{mg} / \mathrm{kg}$. Plasma samples were collected 5, 20, 45, 70, 100, 130, 160, 210, and $360 \mathrm{~min}$ post dose. Efficacy was assessed using digitizing-tablet based Archimedes spirals drawn by patients at multiple time points after administration and washout, as well as wrist-worn uniaxial accelerometers, worn throughout the entire study.

Results: OA plasma levels were already detectable $5 \mathrm{~min}$ after administration, rising to a peak at $100 \mathrm{~min}$, while 1-octanol was only detectable at low levels lacking a dose-response relation. Calculated OA plasma half-life was $88.9 \mathrm{~min}$. Spirography showed a reduction of tremor velocity at spectral peak frequency with a maximum effect starting at 90 min, persisting further until the $360 \mathrm{~min}$ time point. Mean tremor reduction of the right hand in this time period was $39 \%$ with a maximum of $56 \%$. Mean accelerometric tremor severity, calculated based on previously described algorithms (Van Someren, 2006; Nahab, 2007), showed a reduction of $49 \%$ over a 5 -hour period after administration of the study drug.
Conclusions: 1-octanol is effective and safe up to 128 $\mathrm{mg} / \mathrm{kg}$ in ET, with an exponential dose-response curve of OA plasma levels. The effect is most likely mediated through rapid conversion of 1-octanol to OA.

\section{Abstract \#5: Enhancing the Role of the Public in Clinical Research: The Effectiveness of a Multi-Disciplinary Training Program in Increasing Knowledge about the Clinical Research among People with Parkinson's \\ Veronica L. Todaro and Robin Elliott \\ Parkinson's Disease Foundation}

Objective: To assess the potential of a multi-day training program to increase participants capacity, and comfort, in disseminating information about clinical research.

Background: Enhancing the role of the public in the clinical research enterprise, a strategic imperative of the National Institutes of Health, is becoming recognized as a critical variable in building public trust in and support for research, as well as reducing barriers in moving research from bench to bedside.

As a sub-population with interests in the outcomes of clinical research, patients have a special need for the knowledge and skills to effectively serve as representatives with the clinical research enterprise.

In July 2008, the Parkinson's Disease Foundation launched the Clinical Research Learning Institute (CRLI) to address this need within the Parkinson's community. CRLI planning committee members and faculty included members of the Parkinson Study Group, the private sector, and people with Parkinson's.

Design/Methods: A survey was administered to all 24 CRLI participants following each session to assess the increase in knowledge on session content as well as their degree of comfort in disseminating this information to others.

Results: For six of the seven sessions, over $60 \%$ of the participants reported an increase in knowledge of the content presented. In five of the seven sessions, over $50 \%$ of participants reported that their comfort in disseminating information increased.

Conclustions: The majority of CRLI participants saw an increase in knowledge and degree of comfort in speaking on clinical research topics. Further analysis is now underway to identify and assess the opportunities that participants are pursuing in helping to advance clinical research.

\section{Abstract \#6: The Combined Effects of Sciemed Flex ${ }^{\mathrm{TM}}$ and Brain Function Sqautemetry on Memory and Entrapment Neuropathy: A Neuroendocrinologic Concept Study}

\section{Alphonsus Ekwerike \\ Sciencemedicine Research}

Introduction: Sciemed Flex ${ }^{\mathrm{TM}}$ is a neuroendocrinologicosteologic supplementary drug designed to promote mental health, systemic comfort, and joint flexibilities. Its formulatory ingredients are MSM (Methylsulfonalmethane), glucosamine $\mathrm{Hcl}$, Chondroitin, manganese sulfate, Piper methysticum extract, totaling $1235 \mathrm{mg}$ altogether, and Ascorbic acid $6 \mathrm{mg}$ $(100 \% \mathrm{DV})$ as the proprietary blend, with excepients of Calcium phosphate, maltodextrin, cellulose, extra refined vegetable oil, PEG 8000, and glycerol monostearate. Entrapment neuropathy is any mechanically induced irritative/inflammatory disorder that occurs within the neuromuscular anatomical landmarks that clinically manifest tingling, compressive pain, 
muscular weakness, paresthesias, etc. Entrpament neuropathy characterizes neuroendocrinologic deficits due to its compressive-inflammatory affections to the bones, muscles, nerves, blood vessels, and behavioral trends, etc. It obstructs blood flow, electrolytes, and neuropeptic functions, thereby inducing abnormal homeostasis, hypo or hypertonic metabolism, and painful mood swings. BrainFun ${ }^{\mathrm{TM}}$ Squatemetry is a cognitive profiling and therapeutic device that measures the short time, long time, calculative, abstract memories, etc.

Method: A total of 20 normal persons and 60 patients duly diagnosed routinely and with specialists investigations of x-ray, MRI, ECG, EEG, blood samples were studied. Orthopeadic and neurological examinations, respectively, were studied double blindly under four groups of 20 each, namely normasq, Entrap, Entrapflex, and Entrapflexsq. Sciemed flex tab $1 \times 3$ daily was given to the third and fourth groups only. Placebo $1 \times 3$ daily for 30 days was given to the normasq and Entrap groups. BrainFun ${ }^{\mathrm{TM}}$ Sqautemetry and physical therapy $3 \times$ weekly for 30 days applied to all. Altogether this study is for 90 days, with the second 30 days as recovery follow-up period. It is still ongoing. Pain assessment psychometry was used to infer neuralgia status.

Result: The Entrapflexsq group showed quicker recovery rate and better cognitive prospective orientations than all. The Entrapflex group improved more than the Entrap group, while the normasq group showed better cognitive prospective orientation than the Entrap group and measured slightly above Entrapflex.

Conclusion: This result demonstrates that the combination of Sciemed flex tablets, BrainFun ${ }^{\mathrm{TM}}$ Sqautemetry, and physical therapy promote mental health, entrapment release, joint flexibility, and general well being, and shows that pain inhibits cognition.

\section{Abstract \#7: Identifying Potential Subjects for Tourette Syndrome Clinical Research \\ David Shprecher,* Lindsay A. Rubenstein, ${ }^{\dagger}$ Dongwen Wang,* and Roger Kurlan* \\ *University of Rochester; ${ }^{\dagger}$ Boston University School of Medicine}

Objective: To compare two different approaches for identifying appropriate potential subjects for a clinical research study in TS.

Background: We planned to recruit TS patients for a research protocol requiring age range $21-50$ and that subjects be off psychotropic medication for at least six months prior to enrollment.

Methods: Using an electronic billing system, we obtained date of birth for individuals seen in our TS clinic between 1998 and 2000. Based on this, we identified those in the age range for inclusion. We also reviewed paper medical records of all patients seen in our clinic from 2006 through 2008 and identified those in the proper age range. These potentially eligible subjects were then categorized based upon use of psychotropic medication.

Results: Of 128 patients with a diagnosis of TS listed in the electronic billing system, 69 met the criteria for age. Of 328 medical records reviewed, 77 met the criteria for age. Chart review of these 77 cases found that only 8 were not taking psychotropic medication, 48 were taking tic suppressing medication, and 21 were taking psychotropic medication for OCD, ADHD, or depression. Additional potential subjects were found from the family members of 72 reviewed cases. The electronic billing record search required only minutes to complete, while the record review required about 15 hours of effort.

Discussion: Identifying potential subjects from an existing patient pool is a critical step for clinical research study recruitment. The medical record review allowed acquisition of information about medication use and family history that was not available through a query of the electronic billing data. Disadvantages of the chart review might be overcome through use of an electronic database that contains specific information relevant to subject recruitment for research. However, given the reality that no single system may contain all the information needed for clinical research recruitment, a more practical approach will be to integrate the two methods (initial screening using an electronic system followed by chart review to extract more detailed information).

\section{Abstract \#8: Parkinson's Disease Patients Willingness to Accept Risk Associated with Potentially Disease-Modifying Surgical Procedures

\author{
Chandler Gill,* Neil Manus, ${ }^{\dagger}$ Michael Pelster, ${ }^{\dagger}$ \\ James Bryant, ${ }^{\dagger}$ Jason Cook, ${ }^{\dagger}$ and David Charles* \\ *Vanderbilt University Medical Center; ${ }^{\dagger}$ Vanderbilt \\ University
}

Exciting treatments postulated to slow the progression of Parkinson's disease (PD) include gene therapy and deep brain stimulation (DBS). These therapies also have risks of death or permanent disability. If DBS, for example, were proven to be disease modifying, standard treatment would necessitate DBS as early as the diagnosis is reasonably confirmed. Because at this stage patients have little disability, they will choose to receive the therapy only if the magnitude of disease modification is large enough to outweigh the risks. We therefore sought to concretely illuminate the amount of benefit that was perceived by patients to make the risk of DBS reasonable. With IRB exemption, a research assistant was posted in the Vanderbilt Movement Disorders Clinic and interviewed all PD patients over a four-week period. Patients were read a description of a theoretical neuroprotective neurosurgical procedure, including a $10 \%$ risk of infection and $2 \%$ risk of disabling stroke or death. Patients were then asked, if this therapy could delay the amount of time it took them to become disabled or otherwise need significant assistance by $\mathrm{x}$, whether they would want the treatment. The following answer choices were inserted for $\mathrm{x}$ until they answered affirmatively: 6 months, 1, 2, 5, and 10 years. Overall, patients with PD wanted to receive the hypothetical therapy. Only one patient said he would not choose to receive the therapy regardless of how long it extended independence. Of the remaining patients, $50 \%$ said they would choose the therapy with five or fewer years of slowing, and 50\% said they would choose the therapy with greater than five years. The median answer was 6.3 years of additional independence. PD patients would elect a neurosurgical procedure with moderate risks if it offered meaningful disease slowing. Investigators designing future trials of potentially disease-modifying therapies that carry significant risks should consider the likelihood that future patients would choose to receive the therapy, even if the results are positive.

Financial Sponsors: This research was not funded. Vanderbilt University has received income from Medtronic for research lead by Dr. Charles, and he has received income from Medtronic for speaking and consulting services. 